\title{
SELECTED INDICATORS OF THE ROOT QUALITY OF FIFTEEN CULTIVARS OF RED BEET (BETA VULGARIS L.)
}

\author{
Zofia NIZIOŁ-ŁUKASZEWSKA*1, Maria GAWĘDA ${ }^{2}$ \\ ${ }^{1}$ Department of Cosmetology, University of Information Technology and Management in Rzeszow \\ Kielnarowa 386a, 36-020 Tyczyn, Rzeszów, Poland \\ ${ }^{2}$ Department of Vegetable and Medicinal Plants, University of Agriculture in Cracow \\ al. 29 Listopada 54, 31-425 Kraków, Poland \\ Received: August 2014; Accepted: May 2015
}

\begin{abstract}
Evaluation of the quality of 15 cultivars of red beet has been made during four successive vegetative seasons. The study involved cultivars 'Astar $\mathrm{F}_{1}$ ', 'Boro $\mathrm{F}_{1}$ ', 'Ceryl', 'Chrobry', 'Czerwona Kula', 'Egipski', 'Karmazyn', 'Nabab F1', 'Nochowski', 'Opolski', 'Pablo F1', 'Patryk', 'Regulski Cylinder', 'Okrągły Regulski' and 'Rywal'. The mass, diameter of roots, antioxidant activity, the content of dry mass, soluble sugars, betanin and vulgaxanthin in roots were evaluated. The study indicated 'Chrobry' as the cultivar of the most favorable quality features. It was characterized by high antioxidant activity, high content of dry mass, soluble sugars and betalain pigments. Among cultivars of cylindrical shaped roots, content of soluble sugars and antiradical activity was the highest in 'Regulski Cylinder' roots.
\end{abstract}

Key words: red beet, cultivars, antioxidant activity, betalain pigments, soluble sugars

\section{INTRODUCTION}

Red beet is one of the most popular vegetable in Poland. It belongs to the group of 10 vegetables showing the highest antioxidant potential (Vinson et al. 1998; Wettasinghe et al. 2002). Its health benefits are primarily owed to betalaine compounds, which provide protection against reactive forms of oxygen and other free radicals. Due to their natural origin, they contribute to greater protection of human body against carcinogenic substances than their synthetic substitutes (Stintzing \& Carle 2007). These pigments belong to two groups: red-violet betacyanins and yellow betaxanthins. Betacyanins are mainly betanin and izobetanin, while among betaxanthins mainly vulgaxanthins (I and II) can be distinguished (Strack et al. 2003). Betacyanins belong to the group of natural antioxidants, and betanin is commonly called a "rising star among antioxidants" (Nowak et al. 2008). However, no clear connection between the presence of yellow pigments and antioxidant capacity has been observed (Czapski et al. 2009). Betanin present in red beets, marked with $E$ 162 , is also used in the production of natural pigments (Bruch-Kowalska \& Wąsik 1995).

The red beet roots consist mainly of reducing sugars such as glucose and fructose, disaccharides and starch (Zadernowski \& Oszmiański 1994). They are also characterized by a high content of roughage (Wiąckowski 1995) that belongs to the carbohydrates beneficial for the digestive processes.

The purpose of this study was to evaluate the quality of 15 cultivars of red beet based on the content of soluble sugars, dry mass, betalain pigments and antioxidant properties. Also the mass, root diameter and the content of juice in red beets were assessed. 


\section{MATERIALS AND METHODS}

The study was carried out in 2009-2012 in the experimental field of the Department of Vegetable and Medicinal Plants in Mydlniki near Kraków on brown soil. For this study 15 cultivars of red beet from different breeding companies have been rated. Thirteen cultivars, 'Astar $\mathrm{F}_{1}$ ', 'Boro $\mathrm{F}_{1}$ ', 'Ceryl', 'Chrobry', 'Czerwona Kula', 'Egipski', 'Karmazyn', 'Nabab F1', 'Nochowski', 'Opolski', 'Pablo F1', 'Patryk', 'Regulski Cylinder', 'Okrągły Regulski' and 'Rywal' were bred in Poland, and 'Boro $F_{1}$ ' and 'Pablo $F_{1}$ ' - in the Netherland.

Before sowing, seeds were coated with $\mathrm{Fu}-$ naben T. They were sown in rows at intervals of $40 \mathrm{~cm}$ using a seeder. After about 2-4 weeks (depending on the year) thinning was performed at intervals of about $10 \mathrm{~cm}$, so there were 25 plants per square meter. The experimental plots of $6 \mathrm{~m}^{2}$ were arranged in a randomized block with four replications. During the growing season, a mechanical weeding was performed continuously. In the late July and early August a treatment against Cercospora beticola was administered with the use of Curzate fungicide, containing cymoxanil as active ingredient (20 g of the product per 10 liters of water).

The seeds were sown in early July, seedlings were thinned in middle or late July and roots were harvested in October in 2010, and in September in the remaining years.

Immediately after harvest, the root fresh weight and diameter, content of juice and the dry mass by method of Pijanowski at $70{ }^{\circ} \mathrm{C}$ (KrełowskaKułas 1993) were performed. Content of soluble sugars was (\% f.w.) determined by anthrone method (Yemm \& Wills 1954), betanin and vulgaxanthin (mg $\cdot \mathrm{g}^{-1}$ f.w.) according to Nilsson (1970) and the antioxidant activity of the stable radical DPPH (2,2diphenyl-2-picrylhydrazyl) according to Miliauskas et al. 2004.

Al total of 40 representative roots was taken from each cultivar, based on which chemical analysis was performed. Analyses for each cultivar were performed in triplicate. Obtained results were statistically worked out with Statistica 9.0 software, using the analysis of the Tukey HSD test at a level of significance, $\mathrm{p}=0.05$.

\section{RESULTS AND DISCUSSION}

The weather conditions in years 2009 and 2012 differed largely. Extremely adverse climatic events were observed in the second year of the study. These included a great amount of rainfall throughout the whole period of vegetation, especially high in the last ten days of July and August when the field was flooded for some time. The least precipitation was in 2012 . The highest temperatures occurred in July of 2009, 2010 and 2012 and in August 2011. The period from 10 to the 20 July 2010 was the warmest. However, the coolest period was in middle October of the same year (Figs. 1-4). It has been shown that in the year of the highest rainfall (2010) the content of vulgaxanthine and betanine in beet roots was significantly lower compared with 2012, in which there was the least rainfall.

Results from years 2009 and 2012 showed that the largest root diameter had Egipski cultivar, although there were no significant differences between 'Ceryl' and 'Boro $F_{1}$ ' cultivars (Table 1). At the same time, this cultivar was characterized by the lowest antioxidant ability and the lowest content of betanin and vulgaxanthin among examined cultivars (Table 6,7), which is consistent with studies of Watson and Gabelman (1982) who showed that red beets with higher mass contain about $20 \%$ less pigments than those of medium size.

Among the analysed cultivars, 'Nabab $F_{1}$ ' proved to be the least juicy (Table 4). It was also characterized by a low level of sugars and high content of dry mass (Table 3, 5); therefore, it should not be recommended for juice or borscht production. There were no substantial differences in dry mass contents within the examined cultivars (Table 3). The highest content of soluble sugars was found in 'Ceryl', yet it did not vary greatly from the cultivars 'Astar F ', 'Boro F ', 'Chrobry', 'Egipski' and 'Okrągły Regulski'. The lowest content of sugars was noticed in 'Nabab F' ' and 'Karmazyn' (Table 5). In comparison to the study of Elkner et al. (1997), in our experiment 'Czerwona Kula' and 'Chrobry' evidenced a lesser content of dry mass and soluble sugars, which could be a reason of diverse crop conditions. 


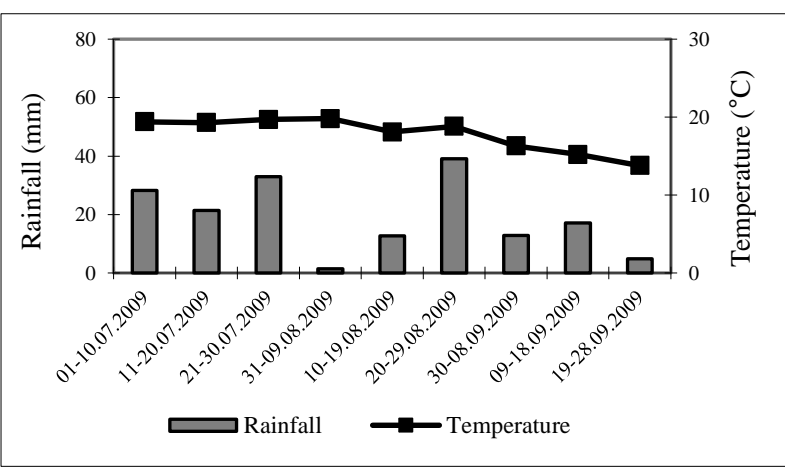

Fig. 1. Average temperatures and rainfall sum in 2009, for periods of 10 days

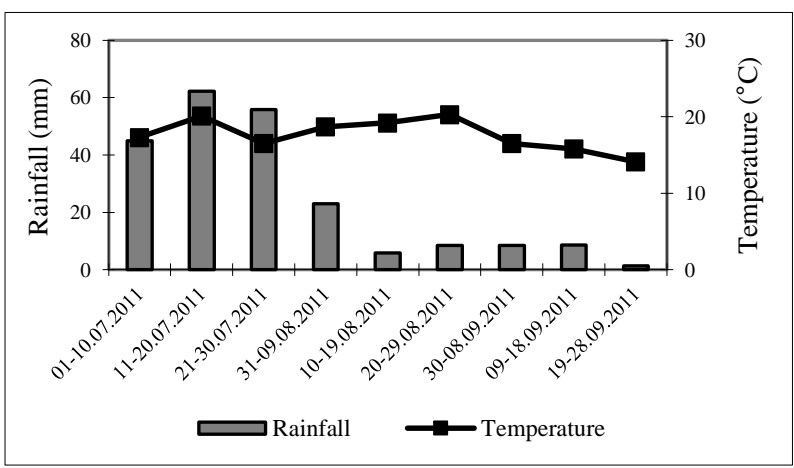

Fig. 3. Average temperatures and rainfall sum in 2011, for periods of 10 days

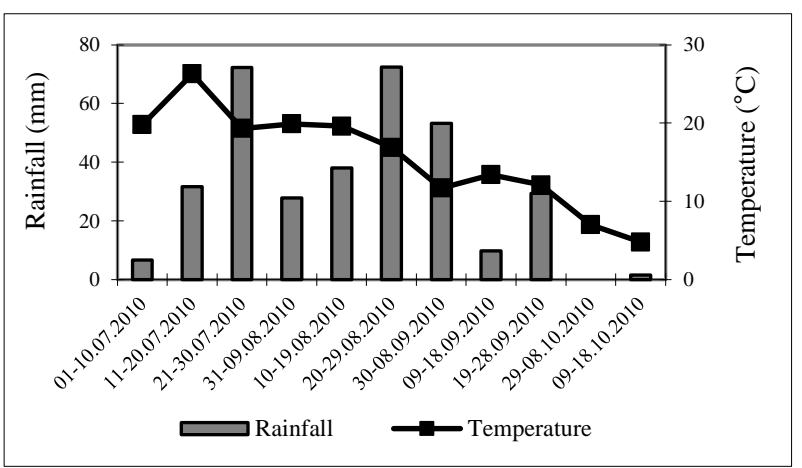

Fig. 2. Average temperatures and rainfall sum in 2010, for periods of 10 days

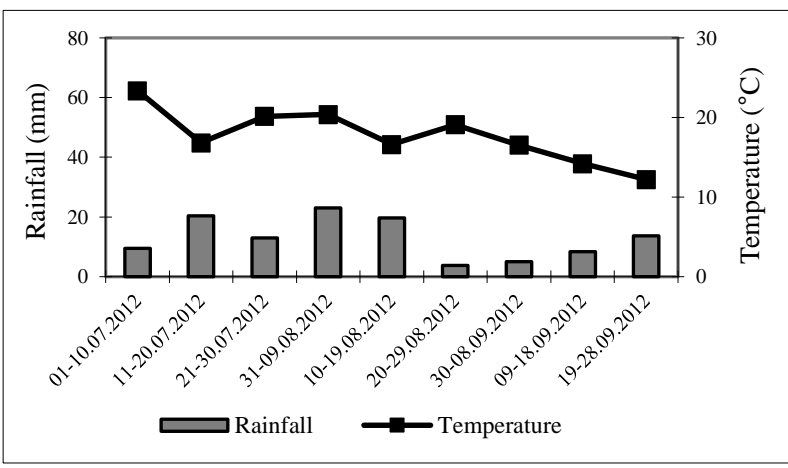

Fig. 4. Average temperatures and rainfall sum in 2012, for periods of 10 days

Table 1. Diameter of roots $(\mathrm{cm})$ of 15 red beet cultivars in 2009-2012

\begin{tabular}{llllll}
\hline \multicolumn{1}{c}{ Cultivar } & 2009 & 2010 & \multicolumn{1}{c}{2011} & \multicolumn{1}{c}{2012} & Mean \\
\hline 'Astar F' & $6.73 \mathrm{c}$ & $6.41 \mathrm{bc}$ & $5.10 \mathrm{abc}$ & $5.50 \mathrm{cde}$ & $5.94 \mathrm{~B}$ \\
'Boro F'' & $7.66 \mathrm{ef}$ & $6.62 \mathrm{c}$ & $5.70 \mathrm{cde}$ & $6.19 \mathrm{de}$ & $6.54 \mathrm{CD}$ \\
'Ceryl' & $7.98 \mathrm{f}$ & $6.42 \mathrm{bc}$ & $6.07 \mathrm{cde}$ & $5.72 \mathrm{cde}$ & $6.55 \mathrm{CD}$ \\
'Chrobry' & $6.83 \mathrm{~cd}$ & $5.88 \mathrm{bc}$ & $5.65 \mathrm{~cd}$ & $5.00 \mathrm{bc}$ & $5.84 \mathrm{~B}$ \\
'Czerwona Kula' & $7.87 \mathrm{f}$ & $5.99 \mathrm{bc}$ & $5.54 \mathrm{bcd}$ & $5.42 \mathrm{bcde}$ & $6.21 \mathrm{BC}$ \\
'Egipski' & $8.96 \mathrm{~g}$ & $8.04 \mathrm{~d}$ & $6.73 \mathrm{e}$ & $5.74 \mathrm{cde}$ & $7.37 \mathrm{D}$ \\
'Karmazyn' & $7.53 \mathrm{ef}$ & $4.03 \mathrm{a}$ & $5.80 \mathrm{cde}$ & $5.67 \mathrm{cde}$ & $5.76 \mathrm{~B}$ \\
'Nabab F'' & $7.49 \mathrm{ef}$ & $5.63 \mathrm{~b}$ & $5.57 \mathrm{~cd}$ & $5.72 \mathrm{cde}$ & $6.10 \mathrm{BC}$ \\
'Nochowski' & $7.30 \mathrm{de}$ & $6.35 \mathrm{bc}$ & $6.21 \mathrm{de}$ & $5.27 \mathrm{bcd}$ & $6.28 \mathrm{BC}$ \\
'Opolski' & $5.12 \mathrm{a}$ & $3.97 \mathrm{a}$ & $4.21 \mathrm{a}$ & $5.28 \mathrm{bcd}$ & $4.65 \mathrm{~A}$ \\
'Pablo F', & $7.42 \mathrm{ef}$ & $6.75 \mathrm{c}$ & $5.84 \mathrm{cde}$ & $3.69 \mathrm{a}$ & $5.93 \mathrm{BC}$ \\
'Patryk' & $7.59 \mathrm{ef}$ & $6.18 \mathrm{bc}$ & $5.71 \mathrm{cde}$ & $6.45 \mathrm{e}$ & $6.48 \mathrm{BC}$ \\
'Regulski Cylinder' & $4.75 \mathrm{a}$ & $4.32 \mathrm{a}$ & $4.25 \mathrm{a}$ & $4.68 \mathrm{abc}$ & $4.50 \mathrm{~A}$ \\
'Regulski Okrągły' & $7.57 \mathrm{ef}$ & $6.03 \mathrm{bc}$ & $6.03 \mathrm{cde}$ & $5.67 \mathrm{cde}$ & $6.32 \mathrm{BC}$ \\
'Rywal' & $6.04 \mathrm{~b}$ & $4.04 \mathrm{a}$ & $4.51 \mathrm{ab}$ & $4.38 \mathrm{ab}$ & $4.74 \mathrm{~A}$ \\
\hline Annual mean & $7.12 \mathrm{~B}$ & $5.78 \mathrm{~A}$ & $5.53 \mathrm{~A}$ & $5.36 \mathrm{~A}$ & 5.95 \\
\hline
\end{tabular}

For $\mathrm{p}=0.05$ (Tukey test), significant differences are marked with different letters 
Table 2. Fresh weight of roots ( $\mathrm{g}$ ) of 15 red beet cultivars in 2009-2012

\begin{tabular}{|c|c|c|c|c|c|}
\hline Cultivar & 2009 & 2010 & 2011 & 2012 & Mean \\
\hline 'Astar $F_{1}^{\prime}$ & $175 \mathrm{ab}$ & 168 cde & $90.2 \mathrm{a}$ & $93.9 \mathrm{abcd}$ & $132 \mathrm{ABC}$ \\
\hline 'Boro $\mathrm{F}_{1}$ ' & $337 \mathrm{bc}$ & $169 \mathrm{cde}$ & $144 \mathrm{bc}$ & $140 \mathrm{~cd}$ & $197 \mathrm{CD}$ \\
\hline ‘Ceryl’' & $239 c$ & 150 abcde & $132 a b c$ & $107 \mathrm{abcd}$ & $157 \mathrm{ABC}$ \\
\hline 'Chrobry' & $237 \mathrm{bc}$ & 129 abcd & $137 \mathrm{abc}$ & $79.5 \mathrm{ab}$ & $145 \mathrm{ABC}$ \\
\hline 'Czerwona Kula' & $279 \mathrm{bc}$ & $134 \mathrm{abcd}$ & $112 \mathrm{abc}$ & $90.1 \mathrm{abc}$ & $154 \mathrm{ABC}$ \\
\hline 'Egipski' & $297 \mathrm{bc}$ & $182 \mathrm{de}$ & $108 \mathrm{ab}$ & $80.4 \mathrm{ab}$ & $167 \mathrm{BC}$ \\
\hline 'Karmazyn' & $234 \mathrm{bc}$ & $106 \mathrm{a}$ & $106 \mathrm{ab}$ & $104 \mathrm{abcd}$ & $137 \mathrm{ABC}$ \\
\hline 'Nabab F & $280 \mathrm{bc}$ & $104 \mathrm{a}$ & $110 \mathrm{ab}$ & $109 \mathrm{bcd}$ & $151 \mathrm{ABC}$ \\
\hline 'Nochowski' & $224 \mathrm{bc}$ & 162 bcde & $142 \mathrm{abc}$ & $75.2 \mathrm{ab}$ & $151 \mathrm{ABC}$ \\
\hline 'Opolski' & $67.8 \mathrm{a}$ & $113 \mathrm{a}$ & $108 \mathrm{ab}$ & $79.6 \mathrm{ab}$ & $92.2 \mathrm{~A}$ \\
\hline 'Pablo $F_{1}$ ' & $567 \mathrm{~d}$ & $200 \mathrm{e}$ & $131 \mathrm{abc}$ & $70.5 \mathrm{ab}$ & $242 \mathrm{D}$ \\
\hline 'Patryk' & $243 \mathrm{bc}$ & $127 \mathrm{abc}$ & $98.7 \mathrm{ab}$ & $145 \mathrm{~d}$ & $154 \mathrm{ABC}$ \\
\hline 'Regulski Cylinder' & $83.7 \mathrm{a}$ & $140 \mathrm{abcd}$ & $137 \mathrm{abc}$ & $57.5 \mathrm{a}$ & $105 \mathrm{AB}$ \\
\hline 'Okrągły Regulski' & $303 \mathrm{c}$ & $117 \mathrm{abc}$ & $119 a b c$ & 108 abcd & $161 \mathrm{BC}$ \\
\hline 'Rywal' & $304 \mathrm{c}$ & $104 \mathrm{a}$ & $163 \mathrm{c}$ & $121 \mathrm{bcd}$ & $173 \mathrm{C}$ \\
\hline Annual mean & $258 \mathrm{~A}$ & $140 \mathrm{~A}$ & $123 \mathrm{~A}$ & $97.2 \mathrm{~A}$ & 155 \\
\hline
\end{tabular}

For $\mathrm{p}=0.05$ (Tukey test), significant differences are marked with different letters

Table 3. Content of dry matter in roots (\%) of 15 red beet cultivars in 2009-2012

\begin{tabular}{llllll}
\hline \multicolumn{1}{c}{ Cultivar } & 2009 & 2010 & 2011 & 2012 & Mean \\
\hline 'Astar F1' & $14.9 \mathrm{gh}$ & $16.6 \mathrm{f}$ & $14.7 \mathrm{ef}$ & $13.6 \mathrm{def}$ & $14.9 \mathrm{~A}$ \\
'Boro F1' & $12.4 \mathrm{a}$ & $16.1 \mathrm{e}$ & $12.8 \mathrm{ab}$ & $12.6 \mathrm{bcd}$ & $13.5 \mathrm{~A}$ \\
'Ceryl' & $14.3 \mathrm{f}$ & $17.6 \mathrm{i}$ & $14.8 \mathrm{fg}$ & $13.5 \mathrm{def}$ & $15.1 \mathrm{~A}$ \\
'Chrobry' & $15.5 \mathrm{j}$ & $16.9 \mathrm{~g}$ & $14.8 \mathrm{efg}$ & $14.0 \mathrm{ef}$ & $15.3 \mathrm{~A}$ \\
'Czerwona Kula' & $13.7 \mathrm{c}$ & $15.8 \mathrm{~d}$ & $14.9 \mathrm{~g}$ & $12.6 \mathrm{bcd}$ & $14.3 \mathrm{~A}$ \\
'Egipski' & $13.6 \mathrm{c}$ & $17.1 \mathrm{~h}$ & $14.6 \mathrm{e}$ & $13.5 \mathrm{def}$ & $14.7 \mathrm{~A}$ \\
'Karmazyn' & $15.0 \mathrm{~h}$ & $15.0 \mathrm{a}$ & $15.6 \mathrm{i}$ & $14.0 \mathrm{ef}$ & $14.9 \mathrm{~A}$ \\
'Nabab F'' & $15.4 \mathrm{i}$ & $17.0 \mathrm{gh}$ & $15.5 \mathrm{i}$ & $13.4 \mathrm{cde}$ & $15.3 \mathrm{~A}$ \\
'Nochowski' & $15.0 \mathrm{~h}$ & $16.9 \mathrm{gh}$ & $14.6 \mathrm{ef}$ & $14.6 \mathrm{f}$ & $15.3 \mathrm{~A}$ \\
'Opolski' & $13.8 \mathrm{~d}$ & $16.7 \mathrm{f}$ & $14.8 \mathrm{efg}$ & $13.4 \mathrm{def}$ & $14.7 \mathrm{~A}$ \\
'Pablo F1' & $12.6 \mathrm{~b}$ & $16.6 \mathrm{f}$ & $13.1 \mathrm{c}$ & $11.5 \mathrm{ab}$ & $13.5 \mathrm{~A}$ \\
'Patryk' & $14.9 \mathrm{f}$ & $17.6 \mathrm{i}$ & $14.3 \mathrm{~d}$ & $11.0 \mathrm{a}$ & $14.4 \mathrm{~A}$ \\
'Regulski Cylinder' & $14.0 \mathrm{e}$ & $15.2 \mathrm{~b}$ & $12.7 \mathrm{a}$ & $13.6 \mathrm{def}$ & $13.9 \mathrm{~A}$ \\
'Regulski Okrągły' & $14.1 \mathrm{e}$ & $18.1 \mathrm{j}$ & $15.2 \mathrm{~h}$ & $12.1 \mathrm{ab}$ & $14.9 \mathrm{~A}$ \\
'Rywal' & $13.6 \mathrm{c}$ & $15.5 \mathrm{c}$ & $13.0 \mathrm{bc}$ & $12.2 \mathrm{bc}$ & $13.6 \mathrm{~A}$ \\
\hline Annual mean & $14.2 \mathrm{~A}$ & $16.6 \mathrm{~B}$ & $14.4 \mathrm{~A}$ & $13.0 \mathrm{~A}$ & 14.5 \\
\hline
\end{tabular}

For $p=0.05$ (Tukey test), significant differences are marked with different letters 
Table 4. Content of juice ( $\mathrm{ml} \cdot \mathrm{kg}^{-1} \mathrm{f} . \mathrm{w}$.) in roots of 15 red beet cultivars in 2009-2012

\begin{tabular}{|c|c|c|c|c|c|}
\hline Cultivar & 2009 & 2010 & 2011 & 2012 & Mean \\
\hline 'Astar $F_{1}$ ' & 460 & 500 & 540 & 300 & 450 \\
\hline 'Boro $F_{1}$ ' & 460 & 540 & 540 & 270 & 453 \\
\hline 'Ceryl' & 570 & 450 & 450 & 240 & 428 \\
\hline 'Chrobry' & 440 & 490 & 490 & 230 & 413 \\
\hline 'Czerwona Kula' & 510 & 450 & 450 & 270 & 420 \\
\hline 'Egipski' & 470 & 490 & 490 & 200 & 413 \\
\hline 'Karmazyn' & 490 & 490 & 480 & 200 & 415 \\
\hline 'Nabab F' & 440 & 370 & 400 & 240 & 363 \\
\hline 'Nochowski' & 500 & 430 & 480 & 220 & 408 \\
\hline 'Opolski' & 510 & 410 & 440 & 280 & 410 \\
\hline 'Pablo $F_{1}$ ' & 550 & 520 & 550 & 200 & 455 \\
\hline 'Patryk' & 580 & 440 & 420 & 300 & 435 \\
\hline 'Regulski Cylinder' & 530 & 550 & 590 & 200 & 468 \\
\hline 'Regulski Okrągły’ & 540 & 380 & 400 & 270 & 398 \\
\hline 'Rywal' & 540 & 470 & 430 & 340 & 445 \\
\hline Annual mean & 506 & 465 & 477 & 251 & 425 \\
\hline
\end{tabular}

For $\mathrm{p}=0.05$ (Tukey test), significant differences are marked with different letters

Table 5. Content of soluble sugars (\% f.w.) in roots of 15 red beet cultivars in 2009-2012

\begin{tabular}{llllll}
\hline \multicolumn{1}{c}{ Cultivar } & \multicolumn{1}{c}{2009} & \multicolumn{1}{c}{2010} & \multicolumn{1}{c}{2011} & \multicolumn{1}{c}{2012} & \multicolumn{1}{c}{ Mean } \\
\hline 'Astar F' & $7.61 \mathrm{de}$ & $7.92 \mathrm{ef}$ & $7.08 \mathrm{efg}$ & $7.31 \mathrm{bcde}$ & $7.48 \mathrm{BCD}$ \\
'Boro F'' & $6.44 \mathrm{ab}$ & $10.17 \mathrm{~g}$ & $6.99 \mathrm{defg}$ & $7.89 \mathrm{def}$ & $7.87 \mathrm{CD}$ \\
'Ceryl' & $8.61 \mathrm{~g}$ & $8.37 \mathrm{f}$ & $8.77 \mathrm{~h}$ & $8.74 \mathrm{f}$ & $8.62 \mathrm{D}$ \\
'Chrobry' & $9.06 \mathrm{~g}$ & $6.75 \mathrm{~cd}$ & $7.75 \mathrm{~g}$ & $7.95 \mathrm{ef}$ & $7.88 \mathrm{CD}$ \\
'Czerwona Kula' & $6.54 \mathrm{ab}$ & $7.25 \mathrm{cde}$ & $7.07 \mathrm{efg}$ & $7.05 \mathrm{bcde}$ & $6.98 \mathrm{BC}$ \\
'Egipski' & $7.35 \mathrm{de}$ & $8.34 \mathrm{f}$ & $7.57 \mathrm{fg}$ & $7.78 \mathrm{cde}$ & $7.76 \mathrm{CD}$ \\
'Karmazyn' & $5.97 \mathrm{a}$ & $7.25 \mathrm{cde}$ & $5.91 \mathrm{~b}$ & $6.44 \mathrm{~b}$ & $6.39 \mathrm{AB}$ \\
'Nabab F1' & $7.33 \mathrm{~d}$ & $5.04 \mathrm{a}$ & $3.19 \mathrm{a}$ & $5.15 \mathrm{a}$ & $5.18 \mathrm{~A}$ \\
'Nochowski' & $9.04 \mathrm{~g}$ & $6.51 \mathrm{bc}$ & $6.88 \mathrm{def}$ & $7.44 \mathrm{cde}$ & $7.47 \mathrm{BC}$ \\
'Opolski' & $8.40 \mathrm{f}$ & $6.41 \mathrm{bc}$ & $6.58 \mathrm{bcde}$ & $6.96 \mathrm{bcd}$ & $7.09 \mathrm{BC}$ \\
'Pablo F', & $7.58 \mathrm{de}$ & $8.54 \mathrm{f}$ & $6.04 \mathrm{bc}$ & $7.18 \mathrm{bcde}$ & $7.34 \mathrm{BC}$ \\
'Patryk' & $6.65 \mathrm{bc}$ & $8.07 \mathrm{ef}$ & $6.15 \mathrm{bcd}$ & $7.19 \mathrm{bcde}$ & $7.01 \mathrm{BC}$ \\
'Regulski Cylinder' & $7.20 \mathrm{~cd}$ & $7.39 \mathrm{de}$ & $6.77 \mathrm{cdef}$ & $7.76 \mathrm{cde}$ & $7.28 \mathrm{BC}$ \\
'Okrągły Regulski' & $8.13 \mathrm{ef}$ & $5.87 \mathrm{ab}$ & $8.89 \mathrm{~h}$ & $7.73 \mathrm{cde}$ & $7.65 \mathrm{CD}$ \\
'Rywal' & $6.41 \mathrm{ab}$ & $7.95 \mathrm{ef}$ & $6.34 \mathrm{bcde}$ & $6.85 \mathrm{bc}$ & $6.89 \mathrm{BC}$ \\
\hline Annual mean & $7.49 \mathrm{~A}$ & $7.46 \mathrm{~A}$ & $6.80 \mathrm{~A}$ & $7.29 \mathrm{~A}$ & 7.26 \\
\hline
\end{tabular}

For $\mathrm{p}=0.05$ (Tukey test), significant differences are marked with different letters 
Table 6. Content of betanin ( $\mathrm{mg} \mathrm{g}^{-1}$ f.w.) in roots of 15 red beet cultivars in 2009-2012

\begin{tabular}{llllll}
\hline \multicolumn{1}{c}{ Cultivar } & \multicolumn{1}{c}{2009} & 2010 & \multicolumn{1}{c}{2011} & \multicolumn{1}{c}{2012} & Mean \\
\hline 'Astar F1' & $0.62 \mathrm{de}$ & $0.64 \mathrm{~b}$ & $1.36 \mathrm{gh}$ & $1.44 \mathrm{def}$ & $1.01 \mathrm{~B}$ \\
'Boro F1' & $0.52 \mathrm{bcd}$ & $0.82 \mathrm{f}$ & $1.07 \mathrm{~cd}$ & $1.13 \mathrm{bc}$ & $0.89 \mathrm{AB}$ \\
'Ceryl' & $0.62 \mathrm{de}$ & $0.92 \mathrm{~g}$ & $1.56 \mathrm{i}$ & $1.65 \mathrm{fg}$ & $1.19 \mathrm{~B}$ \\
'Chrobry' & $0.86 \mathrm{~g}$ & $0.70 \mathrm{c}$ & $1.1 \mathrm{de}$ & $1.22 \mathrm{bcd}$ & $0.97 \mathrm{AB}$ \\
'Czerwona Kula' & $0.47 \mathrm{~b}$ & $0.81 \mathrm{ef}$ & $0.95 \mathrm{bc}$ & $1.06 \mathrm{~b}$ & $0.82 \mathrm{AB}$ \\
'Egipski' & $0.28 \mathrm{a}$ & $0.39 \mathrm{a}$ & $0.63 \mathrm{a}$ & $0.77 \mathrm{a}$ & $0.52 \mathrm{~A}$ \\
'Karmazyn' & $0.82 \mathrm{~g}$ & $0.62 \mathrm{~b}$ & $1.61 \mathrm{i}$ & $1.70 \mathrm{~g}$ & $1.19 \mathrm{~B}$ \\
'Nabab F'' & $0.74 \mathrm{fg}$ & $0.76 \mathrm{de}$ & $1.18 \mathrm{ef}$ & $1.33 \mathrm{cde}$ & $1.00 \mathrm{~B}$ \\
'Nochowski' & $0.78 \mathrm{~g}$ & $0.91 \mathrm{~g}$ & $1.45 \mathrm{~h}$ & $1.56 \mathrm{efg}$ & $1.18 \mathrm{~B}$ \\
'Opolski' & $0.59 \mathrm{cde}$ & $0.77 \mathrm{de}$ & $1.08 \mathrm{de}$ & $1.44 \mathrm{def}$ & $0.97 \mathrm{AB}$ \\
'Pablo F', & $0.62 \mathrm{de}$ & $0.59 \mathrm{~b}$ & $1.08 \mathrm{de}$ & $1.17 \mathrm{bc}$ & $0.86 \mathrm{AB}$ \\
'Patryk' & $0.64 \mathrm{def}$ & $0.79 \mathrm{def}$ & $1.37 \mathrm{gh}$ & $1.19 \mathrm{bc}$ & $1.00 \mathrm{~B}$ \\
'Regulski Cylinder' & $0.65 \mathrm{ef}$ & $0.75 \mathrm{~cd}$ & $0.89 \mathrm{~b}$ & $1.44 \mathrm{def}$ & $0.93 \mathrm{AB}$ \\
'Okrągły Regulski' & $0.59 \mathrm{cde}$ & $0.92 \mathrm{~g}$ & $1.39 \mathrm{~h}$ & $1.00 \mathrm{ab}$ & $0.98 \mathrm{AB}$ \\
'Rywal' & $0.50 \mathrm{bc}$ & $0.77 \mathrm{de}$ & $1.26 \mathrm{fg}$ & $1.35 \mathrm{cde}$ & $0.97 \mathrm{AB}$ \\
\hline Annual mean & $0.62 \mathrm{~A}$ & $0.74 \mathrm{~A}$ & $1.2 \mathrm{~B}$ & $1.3 \mathrm{~B}$ & 0.97 \\
\hline
\end{tabular}

For $\mathrm{p}=0.05$ (Tukey test), significant differences are marked with different letters

Table 7. Content of vulgaxanthin ( $\mathrm{mg} \mathrm{g}^{-1}$ f.w.) in roots of 15 red beet cultivars in 2009-2012

\begin{tabular}{llllll}
\hline \multicolumn{1}{c}{ Cultivar } & \multicolumn{1}{c}{2009} & \multicolumn{1}{c}{2010} & 2011 & \multicolumn{1}{c}{2012} & Mean \\
\hline 'Astar F1' & $0.17 \mathrm{abc}$ & $0.17 \mathrm{a}$ & $0.78 \mathrm{~g}$ & $0.74 \mathrm{efg}$ & $0.47 \mathrm{AB}$ \\
'Boro F1' & $0.17 \mathrm{abc}$ & $0.31 \mathrm{hi}$ & $0.68 \mathrm{def}$ & $0.63 \mathrm{de}$ & $0.45 \mathrm{AB}$ \\
'Ceryl' & $0.18 \mathrm{abc}$ & $0.26 \mathrm{fg}$ & $0.80 \mathrm{gh}$ & $0.73 \mathrm{efg}$ & $0.49 \mathrm{~B}$ \\
'Chrobry' & $0.28 \mathrm{defg}$ & $0.22 \mathrm{cde}$ & $0.63 \mathrm{~d}$ & $0.60 \mathrm{~cd}$ & $0.43 \mathrm{AB}$ \\
'Czerwona Kula' & $0.19 \mathrm{abc}$ & $0.31 \mathrm{hi}$ & $0.44 \mathrm{bc}$ & $0.40 \mathrm{~b}$ & $0.34 \mathrm{AB}$ \\
'Egipski' & $0.13 \mathrm{a}$ & $0.18 \mathrm{ab}$ & $0.18 \mathrm{a}$ & $0.18 \mathrm{a}$ & $0.17 \mathrm{~A}$ \\
'Karmazyn' & $0.29 \mathrm{efg}$ & $0.21 \mathrm{bcd}$ & $0.87 \mathrm{~h}$ & $0.79 \mathrm{fg}$ & $0.54 \mathrm{~B}$ \\
'Nabab F'' & $0.30 \mathrm{fg}$ & $0.36 \mathrm{k}$ & $0.76 \mathrm{fg}$ & $0.72 \mathrm{defg}$ & $0.53 \mathrm{~B}$ \\
'Nochowski' & $0.25 \mathrm{cdefg}$ & $0.29 \mathrm{gh}$ & $0.74 \mathrm{efg}$ & $0.68 \mathrm{def}$ & $0.49 \mathrm{~B}$ \\
'Opolski' & $0.25 \mathrm{cdefg}$ & $0.30 \mathrm{hi}$ & $0.65 \mathrm{de}$ & $0.84 \mathrm{~g}$ & $0.51 \mathrm{~B}$ \\
'Pablo F', & $0.22 \mathrm{bcdef}$ & $0.20 \mathrm{abc}$ & $0.65 \mathrm{de}$ & $0.59 \mathrm{~cd}$ & $0.42 \mathrm{AB}$ \\
'Patryk' & $0.33 \mathrm{~g}$ & $0.35 \mathrm{jk}$ & $0.39 \mathrm{~b}$ & $0.63 \mathrm{de}$ & $0.42 \mathrm{AB}$ \\
'Regulski Cylinder' & $0.20 \mathrm{abcd}$ & $0.24 \mathrm{ef}$ & $0.40 \mathrm{~b}$ & $0.35 \mathrm{~b}$ & $0.30 \mathrm{AB}$ \\
'Okrągły Regulski' & $0.21 \mathrm{abcde}$ & $0.32 \mathrm{ij}$ & $0.88 \mathrm{~h}$ & $0.39 \mathrm{~b}$ & $0.45 \mathrm{AB}$ \\
'Rywal' & $0.15 \mathrm{ab}$ & $0.23 \mathrm{def}$ & $0.51 \mathrm{c}$ & $0.46 \mathrm{bc}$ & $0.34 \mathrm{AB}$ \\
\hline Annual mean & $0.22 \mathrm{~A}$ & $0.26 \mathrm{~A}$ & $0.62 \mathrm{~B}$ & $0.58 \mathrm{~B}$ & 0.42 \\
\hline
\end{tabular}

For $\mathrm{p}=0.05$ (Tukey test), significant differences are marked with different letters 
Table 8. Ratio betanin/vulgaxanthin in roots of 15 red beet cultivars in 2009-2012

\begin{tabular}{llllll}
\hline \multicolumn{1}{c}{ Cultivar } & 2009 & 2010 & 2011 & 2012 & Mean \\
\hline 'Astar F'' & 3.63 & 3.68 & 1.74 & 1.95 & 2.75 \\
'Boro F'' & 3.06 & 2.67 & 1.57 & 1.79 & 2.27 \\
'Ceryl' & 3.43 & 3.57 & 1.95 & 2.26 & 2.80 \\
'Chrobry' & 3.07 & 3.24 & 1.75 & 2.03 & 2.52 \\
'Czerwona Kula' & 2.46 & 2.61 & 2.16 & 2.65 & 2.47 \\
'Egipski' & 2.19 & 2.12 & 3.50 & 4.28 & 3.02 \\
'Karmazyn' & 2.82 & 3.01 & 1.85 & 2.15 & 2.46 \\
'Nabab F'' & 2.48 & 2.12 & 1.55 & 1.85 & 2.00 \\
'Nochowski' & 3.12 & 3.16 & 1.96 & 2.29 & 2.63 \\
'Opolski' & 2.37 & 2.59 & 1.66 & 1.71 & 2.08 \\
'Pablo F' & 2.81 & 2.95 & 1.66 & 1.98 & 2.35 \\
'Patryk' & 1.93 & 2.26 & 3.51 & 1.89 & 2.40 \\
'Regulski Cylinder' & 3.27 & 3.14 & 2.23 & 4.11 & 3.19 \\
'Regulski Okrągły' & 2.83 & 2.87 & 1.58 & 2.56 & 2.46 \\
'Rywal' & 3.32 & 3.29 & 2.47 & 2.93 & 3.00 \\
\hline Annual mean & 2.85 & 2.89 & 2.08 & 2.43 & 2.56 \\
\hline
\end{tabular}

For $\mathrm{p}=0.05$ (Tukey test), significant differences are marked with different letters

Table 9. Antioxidant activity (\% of DPPH scavenging) in roots of 15 red beet cultivars in 2009-2012

\begin{tabular}{lllll}
\hline \multicolumn{1}{c}{ Cultivar } & 2009 & 2010 & \multicolumn{1}{c}{2011} & \multicolumn{1}{c}{ Mean } \\
\hline 'Astar F'' & $23.4 \mathrm{~b}$ & $49.5 \mathrm{i}$ & $36.3 \mathrm{ef}$ & $36.4 \mathrm{GH}$ \\
'Boro F1' & $26.2 \mathrm{bcd}$ & $31.0 \mathrm{def}$ & $29.4 \mathrm{cde}$ & $28.9 \mathrm{CDEFG}$ \\
'Ceryl' & $30.4 \mathrm{e}$ & $23.0 \mathrm{abc}$ & $31.8 \mathrm{de}$ & $28.4 \mathrm{CDEFG}$ \\
'Chrobry' & $36.1 \mathrm{f}$ & $32.1 \mathrm{efg}$ & $32.6 \mathrm{de}$ & $33.6 \mathrm{FGH}$ \\
'Czerwona Kula' & $25.9 \mathrm{bcd}$ & $16.5 \mathrm{a}$ & $27.5 \mathrm{bcd}$ & $23.3 \mathrm{ABCD}$ \\
'Egipski' & $14.6 \mathrm{a}$ & $18.4 \mathrm{ab}$ & $17.8 \mathrm{a}$ & $16.9 \mathrm{~A}$ \\
'Karmazyn' & $31.4 \mathrm{e}$ & $24.2 \mathrm{bcd}$ & $28.6 \mathrm{bcde}$ & $28.1 \mathrm{BCDEFG}$ \\
'Nabab F'' & $28.7 \mathrm{de}$ & $34.2 \mathrm{fg}$ & $33.5 \mathrm{de}$ & $32.1 \mathrm{EFGH}$ \\
'Nochowski' & $32.0 \mathrm{e}$ & $44.2 \mathrm{hi}$ & $43.4 \mathrm{f}$ & $39.9 \mathrm{H}$ \\
'Opolski' & $24.0 \mathrm{bc}$ & $19.8 \mathrm{abc}$ & $21.6 \mathrm{abc}$ & $21.8 \mathrm{ABC}$ \\
'Pablo F1' & $15.8 \mathrm{a}$ & $35.3 \mathrm{fg}$ & $23.1 \mathrm{abc}$ & $24.7 \mathrm{ABCDE}$ \\
'Patryk' & $28.0 \mathrm{cde}$ & $36.9 \mathrm{fg}$ & $33.2 \mathrm{de}$ & $32.7 \mathrm{EFGH}$ \\
'Regulski Cylinder' & $25.6 \mathrm{bcd}$ & $38.1 \mathrm{gh}$ & $25.3 \mathrm{abcd}$ & $29.7 \mathrm{CDEFG}$ \\
'Okrągły Regulski' & $30.5 \mathrm{e}$ & $25.7 \mathrm{cde}$ & $28.3 \mathrm{bcde}$ & $28.2 \mathrm{BCDEFG}$ \\
'Rywal' & $23.9 \mathrm{bc}$ & $16.3 \mathrm{a}$ & $20.4 \mathrm{ab}$ & $20.2 \mathrm{AB}$ \\
\hline Annual mean & $26.4 \mathrm{~A}$ & $29.7 \mathrm{~A}$ & $28.9 \mathrm{~A}$ & 28.3 \\
\hline
\end{tabular}

For $\mathrm{p}=0.05$ (Tukey test), significant differences are marked with different letters 
A content of betalain pigments depends on the genetic predispositions and conditions during cultivation, especially on the weather conditions (Krężel 2005). In our experiment, due to the smallest amount of rainfall in 2012 the roots had the lowest mass, diameter, and content of dry weight; however, they contained the largest amount of betalain pigments (Table 6). Temperature is one of the most important features impacting the color of red beets. Hot, dry summers, and temperatures above $25{ }^{\circ} \mathrm{C}$ negatively affect the betalain pigments content (Magruder 1941; Lorenz 1947; Bradley \& Dyck 1967) which is in agreement with the results of Niziol-Łukaszewska and Gawęda (2014). High temperatures in the first three weeks of cultivation caused a drastic fall in the content of betacyanin and betaxanthin. In 2010, when the temperature was over $25^{\circ} \mathrm{C}$ the very low levels of betalain pigments were recorded.

At present, well colored red beets with a high content of pigments are desired by farmers. According to Biegańska-Marecik et al. (2007), 'Chrobry' and 'Nochowski' meet that expectation. Study of Mikołajczyk and Czapski (2006) proved that the contents of red pigment were $220 \mathrm{mg} \cdot 100 \mathrm{ml}^{-1}$ and $187.2 \mathrm{mg} \cdot 100 \mathrm{ml}^{-1}$ in 'Chrobry' and 'Nochowski', respectively. In this study the content of red pigment in these cultivars was lower. The highest contents were recorded in 'Ceryl' and 'Karmazyn' although the significance between other cultivars was not stated. The exception was 'Egipski' in which the average content of betanin was $40 \%$ lower in comparison to the cultivars richest with this ingredient. Similar results were obtained by Sobkowska et al. (1991), who reported that 'Egipski' contained approximately half the amount of pigment as compared to other cultivars analysed and 'Czerwona Kula' even less. In our study, 'Czerwona Kula' contained more red pigments than 'Egipski'.

The examination of the content of yellow pigments in red beets undertaken by Mikołajczyk and Czapski (2006) who demonstrated that the content of vulgaxanthin for 'Chrobry' was $125.7 \mathrm{mg} \cdot 100 \mathrm{ml}^{-1}$, whereas for 'Nochowski' it was $80.2 \mathrm{mg} \cdot 100 \mathrm{ml}^{-1}$.
According to Elkner et al. (1997), the quantity of vulgaxanthin in 'Chrobry' was $84.5 \mathrm{mg} \cdot 100 \mathrm{~g}^{-1}$ of juice. The results of our experiment indicated a lower content of vulgaxanthin in the listed cultivars, as the lowest average amount of vulgaxanthin was detected in 'Egipski', although it was not significantly different from the other nine cultivars (Table 7). Low content of pigments in 'Egipski' did not unfavorably influence the average betanin : vulgaxanthin ratio. A similar ratio of pigments in 'Egipski' was noticed by Sobkowska et al. (1991). This study found a high content of vulgaxanthin in 'Karmazyn' and 'Nabab F'. Moreover, 'Nabab F1' had the lowest betanin/vulgaxanthin ratio among the examined cultivars. The highest betanin/vulgaxanthin ratio was observed in 'Regulski Cylinder', 'Rywal' and 'Egipski' (Table 8).

In recent years, the interest in health-promoting compounds has increased greatly because of the high risk for lifestyle-related diseases. It concerns mainly the phenolic group present in betalaines, which is responsible for antioxidant properties of red beets (Grajek 2007). This study shows that 'Egipski' was characterized by the lowest antioxidant activity, and the highest ability for scavenging free radicals was found in 'Nochowski' although there were no significant differences with other four cultivars (Table 9). On the basis of the research conducted by Gębczyński (2005) it was stated that the antioxidant activity for 'Czerwona Kula' was $56.7 \%$, nonetheless, in our experiment this capacity was about half as much and amounted to $23.36 \%$.

\section{CONCLUSIONS}

1. Red beet 'Chrobry' is characterized by the roots of a high quality and high contents of health-promoting compounds as dry matter, sugars and betalains and has a high antioxidant activity

2. Among cultivars of cylindrical shape of roots ('Rywal', 'Opolski', 'Regulski Cylinder'), 'Regulski Cylinder' was characterized by the highest content of soluble sugars and also the highest antioxidant activity. 
3. Antioxidant activity and also the content of betanin and vulgaxanthin was the lowest in cultivar 'Egipski', which was characterized by a large content of soluble sugars, high fresh weight and diameter as well as high betanin/vulgaxanthin ratio.

\section{REFERENCES}

Biegańska-Marecik R., Czapski J., Błaszczyk P. 2007. Determination of cultivar and technological process effect on the occurrence of bitter taste in red beet. Food. Science. Technology. Quality 3(52): 62-70. [in Polish with English abstract]

Bradley G.A., Dyck R.L. 1967. Yield and quality of table beets. Arkansas Farm Research 16(4): 9.

Bruch-Kowalska M., Wąsik A. 1995. Betanina /E-162/ naturalny barwnik spożywczy z buraków ćwikłowych. Conference Proceedings: Jakość surowca warzywnego do przetwórstwa. Research Institute of Vegetable Crops, Skierniewice 19-20 October, pp. 133-135. [in Polish]

Czapski J., Mikołajczyk K., Kaczmarek M. 2009. Relationship between antioxidant capacity of red beet juice and contents of its betalain pigments. Polish Journal of Food and Nutrition Sciences 59(2): 119-122.

Elkner K., Badełek E., Adamicki F. 1997. The effect of cultivar and storage conditions on quality of red beet. Bulletin of Vegetable Crops Research Work 46: 67-78. [in Polish with English abstract]

Gębczyński P. 2005. Content of antioxidative constituents in fresh, cooked and frozen red beet. Bromatologia i Chemia Toksykologiczna 38: 335-341. [in Polish with English abstract]

Grajek W. 2007. Przeciwutleniacze w żywności. Aspekty zdrowotne, technologiczne, molekularne i analityczne. WNT, Warszawa, pp. 325-368. [in Polish]

Krełowska-Kułas M. 1993. Badanie jakości produktów spożywczych. PWE, Warszawa, pp. 125-198. [in Polish]

Krężel J. 2005. Usefulness of selected cultivars of beetroot for the cultivation for bunch-harvest for store and processing. Scientific Journal of Wrocław University of Environmental and Life Sciences, Agronomy 515: 325-332. [in Polish with English abstract]

Lorenz O.A. 1947. The effect of certain planting and harvest dates on the quality of table beets. Proceedings of the American Society for Horticultural Science 49: 270-274.

Magruder R. 1941. Growing conditions have important effect on beet and carrot pigmentation. Canning Age 22: 93.

Miliauskas G., Venskutonis P.R., van Beek T.A. 2004. Screening of radical scavenging activity of some medicinal and aromatic plant extracts. Food Chemistry 85: 231-237. DOI: 10.1016/j.foodchem.2003.05.007.

Mikołajczyk K., Czapski J. 2006. The antioxidant activity and content of betalain pigments in different variety of red beet. Bromatologia i Chemia Toksykologiczna. Suppl. 39: 437-441. [in Polish with English abstract]

Nilsson T. 1970. Studies into the pigments in beetroot (Beta vulgaris ssp. vulgaris var. rubra L.). Lantbrukshögskolans Annaler 36: 179-219.

Nizioł-Łukaszewska Z., Gawęda M. 2014. Changes in quality of selected red beet (Beta vulgaris L.) cultivars during the growing season. Folia Horticulturae 26(2):139-146. DOI: 10.1515/fhort-2015-0005.

Nowak D., Kidoń M., Syta M. 2008. Assessing changes in antioxidant activity of dried red beet and celeriac depending on individual operations applied. Food. Science. Technology. Quality 4(59): 227-235.

Stintzing F.C., Carle R. 2007. Betalains - emerging prospects for food scientists. Trends in Food Science \& Technology 18: 514-525. DOI: 10.1016/j.tifs.2007.04.012.

Sobkowska E., Kaczmarek R., Czapski J., Sobiech B., Sikorski K. 1991. Czynniki wpływające na jakość buraka ćwikłowego jako surowca w przetwórstwie i do produkcji barwników. Przemysł Fermentacyjny i Owocowo-Warzywny 35(2): 18-21. [in Polish]

Strack D., Vogt T., Schliemann W. 2003. Recent advances in betalain research. Phytochemistry 62: 247-269. DOI: 10.1016/s0031-9422(02)00564-2.

Vinson J.A., Hao Y., Su. X., Zubik L. 1998. Phenol antioxidant quantity and quality in foods: vegetables. Journal of Agricultural and Food Chemistry 46: 3630-3634. DOI: 10.1021/jf980295o.

Watson J.F., Gabelman W.H. 1982. Seasonal changes and cultivar differences in pigment concentrations and percent dissolved solids in roots of table beets. Journal of the American Society for Horticultural Science 107(5): 713-716. 
Wiąckowski S.K. 1995. Próba ekologicznej oceny żywienia, żywności i składników pokarmowych. PWN, Warszawa, pp. 67-81. [in Polish]

Wettasinghe M., Bolling B., Plhak L., Parkin K. 2002. Screening for phase II enzyme-inducing and antioxidant activities of common vegetables. Journal of Food Science 67: 2583-2588. DOI: 10.1111/j.1365-2621.2002.tb08781.x.

Zadernowski R., Oszmiański J. 1994. Wybrane zagadnienia z przetwórstwa owoców i warzyw. ART, Olsztyn, pp. 10-16. [in Polish]

Yemm E.W., Wills A.J. 1954. The estimation of carbohydrates in plant extracts by anthrone. Biochemical Journal 57: 508-514. DOI: 10.1042/bj0570508. 